\title{
FSO Communication: Benefits, Challenges, and its Analysis in DWDM Communication System
}

\author{
Haleema Khalil ${ }^{1}$, Farhan Qamar $^{2}$, Romana Shahzadi ${ }^{3}$, Mudassar Ali ${ }^{4}$, M. Faisal Nadeem ${ }^{5}$, Nouman \\ Qamar $^{6}$, Intisar Ali Sajjad ${ }^{7}$ \\ (Received March 07, 2020; Revised March 25, 2020; Accepted April 01, 2020) \\ Online First Publication \\ DOI: $10.33317 /$ ssurj.181
}

\begin{abstract}
Free-space optical (FSO) communication is one of the choices of researchers for most of the bandwidth hungry applications in evolving networks where the deployment of optical fiber is not directly possible as a transmission medium. In this research article, benefits, challenges, applications, and role of FSO is discussed in detail for evolving networks. Further, performance of FSO communication system is tested using four channels of dense wavelength division multiplexing (DWDM). Various simulations are performed on FSO including different weather conditions, that directly affect the link performance. Many important parameters such as distance, data rate, bit error rate, transmitter power, and attenuation under different weather conditions are tested in this research work. The operation of FSO communication system is carried out in the range $760-850 \mathrm{~nm}$ where equal channel spacing is considered for the working of DWDM communication system. Moreover, a fair comparison of proposed system is also presented for its operation in two more bands i.e. $C$ and L-band, to show which one offers better performance. Simulation are performed in Optisystem 14.0 and MATLAB. For the analysis of proposed system, results are presented in the form of BER and Q-factor plots.
\end{abstract}

Index Terms - Dense Wavelength Division Multiplexing, Free Space Optics, Weather Effects, Q-Factors

\section{INTRODUCTION}

The exponential increase in the bandwidth requires technology that should lead beyond the conventional copper wires and meet the demand of increasing bandwidth. This urge of high data rate and fast speed of wireless technology resulted in the emergence of FSO [1]. Such systems are helpful in establishing integration of optical and wireless networks using minimum power and cost to provide a compact and simple solution.

With the passage of time, as the demands on deliverable data rates tend to increase, the conventional RF technologies remain far behind than the current requirements. Although optical fiber backhaul links offer high data rates, yet the initial investment and deployment cost are important to consider. Moreover, optical links limit their applications in remote areas as their deployment is not feasible in such areas. Recently, the FSO systems appear as an alternative for backhaul next- generation links. FSO having inherent attributes has been an attractive resource due to its potential for providing wireless services. Optical wireless systems such as FSO provide large data rate solutions to bandwidth dependent applications. However, weather constraints on such technologies pose a great challenge

to their deployment in the areas where high reliability cannot be tolerated due to their adverse effects [2], [3].

\section{A. Abbreviations}

The abbreviations used are shown in Table I, below.

Table I: Abbreviations

\begin{tabular}{|l|l|}
\hline DWDM & Dense Wavelength Division Multiplexing \\
\hline FSO & Free Space Optics \\
\hline OFC & Optical Fiber Communication \\
\hline BER & Bit Error Rate \\
\hline CW Laser & Continuous Wave Laser \\
\hline OSA & Optical Spectrum Analyzer \\
\hline OA & Optical Amplifier \\
\hline PAT & Pointing, Acquisition \& Tracking \\
\hline LOS & Line of Sight \\
\hline RO-FSO & Radio over Free Space Optics \\
\hline CO-OFDM & Coherent Optical Orthogonal Frequency Division \\
& Multiplexing \\
\hline DB & Duo-binary \\
\hline OOK & On-Off Keying \\
\hline DP-16QAM & Dual Polarized 16 QAM \\
\hline PPM & Pulse-Position Modulation \\
\hline LM & Link Margin \\
\hline MUX & Multiplexer \\
\hline DEMUX & Demultiplexer \\
\hline LPF & Low Pass Filter \\
\hline PRBS & Pseudo-Random Bit Sequence Generator \\
\hline
\end{tabular}

\section{B. What are FSO Systems? How do They Work?}

FSO systems are also named as open-air photonics, free-space photonics, optical wireless technology, or infrared broadband technology. These systems operate in the near-infrared region wavelength ranging from $399-352.6 \mathrm{THz}$ and between 199.8 -

${ }^{1}$ Designation, Department of FT \& IE, University of Engineering and Technology, Taxila, Pakistan. haleemakhali1932@gmail.com

${ }^{2}$ Designation, Department of FT \& IE, University of Engineering and Technology, Taxila, Pakistan. farhan.qamar@uettaxila.edu.pk

${ }^{3}$ Designation, Department of FT \& IE, University of Engineering and Technology, Taxila, Pakistan. romana.farhan@uettaxila.edu.pk

${ }^{4}$ Designation, Department of FT \& IE, University of Engineering and Technology, Taxila, Pakistan. mudassar.ali @ hotmail.com

${ }^{5}$ Designation, 2Department of Electrical Engineering, University of Engineering and Technology, Taxila, Pakistan. faisal.nadeem@uettaxila.edu.pk

${ }^{6}$ Designation, 2Department of Electrical Engineering, University of Engineering and Technology, Taxila, Pakistan. nouman.qamar@uettaxila.edu.pk

${ }^{7}$ Designation, 2Department of Electrical Engineering, University of Engineering and Technology, Taxila, Pakistan. intisar.ali@uettaxila.edu.pk 
187.3 THz. The way in which FSO communicates is like optical fiber, however, it connects a pair of photodetector transceivers through a laser beam and uses free space medium for the propagation of light. Line of sight optical links have great potential to serve huge data transmissions and to establish seamless connectivity [4], [5].

FSO serves in an unlicensed band in which an effective point to point communication is made. Atmospheric conditions such as rain, fog, haze affect the link quality and signal propagation [6]. Thus, the weather conditions determine the reliability of FSO communication system greatly.

\section{Role of FSO in Current Wireless Communication}

In FSO, the air is used as transmission media in which transmission is greatly affected by different weather phenomena. FSO systems are severely disturbed by the atmospheric effects and attenuation under different weather conditions which vary due to the temperature and pressure of the atmosphere, in which signals travel. The attenuation occurs in FSO as the atmospheric channel is not ideal. Therefore, we can get high BER, scattering and absorption effects of optical beams thus, degrading the system performance severely. The most critical weather conditions are snow, rain, fog and clouds which result in huge scattering of signal. In these systems, when the power density of the beam decreases, the system becomes unavailable. Therefore, to maintain appropriate power levels, weather effects should be considered. Furthermore, using the concept of spatial diversity i.e. using multiple laser beams, the availability of these system can be improved.

The quality of transmission is monitored by realizing the BER. BER increases as the transmission power decreases and attenuation increases. It has been found that forward error correction techniques help to attain good Q-factor values and lowest BER for FSO communication. FSO transceivers operate in the same way as for optical fiber communication systems. However, as the medium of signal propagation is air, so due to multiple factors, path loss is higher in these systems. Transmission distance, weather, data rate, laser wavelength, scattering, scintillation, absorption pointing error effects are some of the major elements that count to system overall performance [7].

To effectively utilize the bandwidth of FSO channel, DWDM is one of the promising solutions. DWDM enhances system capacity by increasing total no of channels/frequencies and thus, reducing cost of system by using only one FSO channel. This paper utilizes DWDM based FSO communication to effectively utilize the bandwidth in the cases where direct termination of optical fiber is either not possible or very difficult.

\section{Advantages of FSO Communication}

In FSO system, a low power modulated beam transmits the data stream using air as a medium. FSO is viable solution for high data rate and voice transmission. It is significantly used as an alternative to solve bottleneck connectivity issues and a replacement for RF/Microwave links. FSO systems are helpful in providing small size, lightweight, high bandwidth, and low power cost alternatives to microwave solutions. To attain high level benefits in comparison to optical fiber and microwave systems, FSO makes use of high frequencies that enables these systems to support enhanced security, capacity, and higher data rates [4]. FSO link is 25 times more efficient than RF link in terms of capacity as it is immune to EM waves thus, ensures broadband communication. Some additional benefits include quick installation, license free service, short time deployment, low BER, wiretapping safety and high security due to directional and narrow beam. The narrow beam makes it difficult to detect, intercept and jamming.

FSO can be used to provide last-mile connectivity. It can help in disaster recovery and broadband access to remote areas. These systems can be deployed in next-generation optical links for point or multi-point solutions. Its deployment cost is less than an optical fiber or microwave link as it takes only one-fifth of the cost of fiber layout [5], [6]. It can also transfer broadband services on point to point links efficiently. Although FSO has many advantages however, LOS is essential parameter which should be taken into account for the seamless connection [8].

\section{E. Challenges in FSO Communication}

One of the major problems that arises in FSO deployment is link availability which greatly depends on the reliability of equipment and infrastructure design. Despite having great potential for next-generation access uses, widespread deployment of FSO is vulnerable to availability due to atmospheric variations as weather attenuation affects the FSO link performance severely. It results in the degradation of received signal power which can impair the operation of receiver side. An additional challenge can be seen when extreme PAT accuracies are mandatory for establishing a link between mobile terminals using FSO [9]. The deployment of FSO link in hilly areas is also a big challenge due to snow and fog conditions.

While deploying a robust FSO link, designers should consider some specific parameters associated with the link performance. For example, scattering is caused by fog, snow and rain. Scattering deflects away a portion of signal traveling from the source to receiver. Random changes in the refractive index of air cause turbulence which results in random phase at optical receiver. Moreover, mechanical misalignment or error in tracking systems of FSO links can badly affect performance. To achieve acceptable performance of FSO link, transmitter's optimization plays an important role [10]. Optimization of transmitter includes accurate modulation technique, suitable light source, transmission wavelength and appropriate transmitting power level [11].

\section{F. FSO Communication Vs Optical Fiber}

In an optical fiber, the light pulses carry the information from one point to another using glass medium whereas in FSO systems, air is used as medium and light source transfers the data stream through it. FSO is termed as communication at the speed of light because light travels faster in air than in the glass. The channel radiation remains confined in the waveguide medium in an optical fiber, whereas in FSO, diffraction dominates as the radiations propagate away from the source. However, in both the cases modulated laser light is used to transmit the data. 
One of the big differences between FSO and optical fiber is attenuation which significantly limits the distances and data rate in FSO communication as compared to optical fiber communication. The attenuation in optical fiber is very less as compared to FSO system. It is just $0.2 \mathrm{~dB} / \mathrm{km}$ in commercially available single mode optical fiber whereas attenuation in FSO communication starts from $6 \mathrm{~dB} / \mathrm{km}$ for normal weather and increases greatly for more severe weathers or atmospheric conditions.

FSO systems can be mounted on the roofs top or buildings. It consists of an optical transceiver which allow its transmission or reception in full-duplex mode. It contains a high-power transceiver and a lens that transmits light through air to another receiving lens. If the transmitter does not produce enough parallel beam to travel up to a distance, the collimation can be done with the help of lenses. The lens connects to a high sensitivity receiver through optical fiber [11]. Also, FSO system can be coherent and non-coherent based on the detection technique. These systems are easy to implement and quite flexible as any type of modulation technique i.e. amplitude, phase or frequency can be used. On the other hand, deploying optical fiber is a time taking and hectic process which requires measurement of sites, proper routes and lot of initial investments. In short, FSO has an edge over optical fiber due to its flexible service, less expense and fast deployment.

\section{G. Applications}

FSO systems are widely used in military applications because of the benefit of their use for greater than $1 \mathrm{~km}$. In the configuration of three or more laser working together, larger distances can be achieved. Technically, FSO can be used in optical links for different types of military communication e.g. ship to ship, building to building, satellite to ground and aircraft to ground communications [8].

It can also be deployed for connecting a network and as an extension for the metro rings. It can support enterprise applications due to its flexibility i.e. connectivity to short-area networks and from local area network to local area network. Further, it can be used in access applications e.g. gigabit ethernet access. In case of any failure, it is an alternative for supporting backup link for fiber [12].

\section{H. Literature Review}

The demand for high-speed access is increasing gradually and each infrastructure requires high data rates to meet the user demands. Owing to higher bandwidth demands, it has become a challenge for the researchers to ensure the security of data. Due of the short distances supported by the FSO as compared to optical fiber it is easy to manage security features in FSO communication. Thus, FSO can be essentially helpful where security, performance and cost-effective solutions are required at the same time.

In [1], the experiments are performed for a $16 \times 2.5 \mathrm{~Gb} / \mathrm{s}$ FSO system to analyze critical weather conditions using RZ format. The system performance degrades drastically as the attenuation increases due to scattering, absorption, free space path loss and other losses. It is found that when attenuation increases due to severe weathers, the Q-factor and eye height decreases gradually. The proposed system supports very limited distances.

The simulation results for the FSO system at different parameters such as high distance, bit rate, transmitting power and wavelength are evaluated in [2]. In outdoor communication, atmospheric attenuation is a critical factor that affects the quality of the signal. Dense fog has the highest attenuation while clear weather conditions gave the best results for the propagation of the signal.

The analysis of WDM in FSO communication using independent eight channels to carry data under clear weather conditions is monitored in [10] by varying the beam divergence. Beam divergence plays an important role in atmospheric turbulences. The results have shown that even a small improvement in beam divergence of $1 \mathrm{mrad}$ can offer a significant improvement to the link performance. So, when the beam divergence decreases, the link will work for a prolonged distance.

A numerical investigation and simulation modeling of the WDM FSO system has been performed in [13] and shows factors that affect the quality of data transmission. It was deduced that the performance of FSO is highly affected by rain and haze conditions. Also, the FSO wavelength of 1550 $\mathrm{nm}$ produces less effect in atmospheric attenuation. A short link range and low data rate can optimize the transmission components of the FSO system.

The effects of bad weather conditions on the FSO link have been analyzed in [13]. Power spectrums at different wavelength windows of $\mathrm{S}, \mathrm{C}$ and $\mathrm{L}$ band are visualized so that suitable WDM system can be designed based on FSO. It has been observed that under low attenuation values, all three bands can be used simultaneously as all of them have low attenuation bands. S-Band is not preferable for high attenuation condition .C and L band show comparable performance at high attenuation, so they can use in conjugation. A summarized literature review is shown in Table II.

\begin{tabular}{|c|c|c|c|c|c|c|c|}
\hline Ref & Objective & $\begin{array}{c}\text { Modulatio } \\
\text { n } \\
\text { Formats/ } \\
\text { Simulatio } \\
\text { n Model }\end{array}$ & WDM & $\begin{array}{l}\text { Weat } \\
\text { her } \\
\text { Condi } \\
\text { tions }\end{array}$ & $\begin{array}{l}\text { Q- } \\
\text { Fact } \\
\text { or }\end{array}$ & BER & $\begin{array}{c}\text { Spati } \\
\text { al } \\
\text { Diver } \\
\text { sity }\end{array}$ \\
\hline$[1]$ & $\begin{array}{c}\text { Effect of } 16 \text { channel } \\
\text { FSO in critical weather } \\
\text { conditions } \\
\end{array}$ & RZ & $\sqrt{ }$ & $\sqrt{ }$ & $\sqrt{ }$ & $\sqrt{ }$ & \\
\hline [3] & $\begin{array}{c}\text { To increase reliability } \\
\text { of link }\end{array}$ & $\begin{array}{l}\text { Hybrid } \\
\text { RF/FSO }\end{array}$ & & & $\sqrt{ }$ & $\sqrt{ }$ & $\sqrt{ }$ \\
\hline [2] & $\begin{array}{c}\text { Flow detection in } \\
\text { optical wireless DCNs }\end{array}$ & NRZ & $\sqrt{ }$ & & & & \\
\hline$[4]$ & $\begin{array}{c}\text { Visibility range effect } \\
\text { on optical } \\
\text { communication } \\
\text { attenuation } \\
\end{array}$ & $\begin{array}{l}\text { Monte } \\
\text { Carlo } \\
\text { simulation }\end{array}$ & & $\sqrt{ }$ & & & \\
\hline [5] & $\begin{array}{l}\text { Weather effects on } \\
\text { hybrid FSO/RF link }\end{array}$ & OOK & & $\sqrt{ }$ & & $\sqrt{ }$ & \\
\hline [6] & $\begin{array}{c}\text { Weather attenuation } \\
\text { effects of FSO link } \\
\text { performance }\end{array}$ & $\begin{array}{l}\text { Weather } \\
\text { attenuation } \\
\text { model }\end{array}$ & & $\sqrt{ }$ & & & \\
\hline [7] & Multi-hop FSO network & $\begin{array}{l}\text { PPM, } \\
\text { OOK }\end{array}$ & & $\sqrt{ }$ & & $\sqrt{ }$ & \\
\hline [8] & $\begin{array}{l}\text { Comparative Analysis } \\
\text { of Point to Point FSO }\end{array}$ & $\begin{array}{l}\text { FSO } \\
\text { WDM }\end{array}$ & $\sqrt{ }$ & $\sqrt{ }$ & $\sqrt{ }$ & $\sqrt{ }$ & \\
\hline
\end{tabular}




\begin{tabular}{|c|c|c|c|c|c|c|c|}
\hline & System & model & & & & & \\
\hline [9] & $\begin{array}{c}\text { Error in FSO } \\
\text { communication system }\end{array}$ & $\begin{array}{l}\text { Pointing } \\
\text { error } \\
\text { model }\end{array}$ & & $\sqrt{ }$ & & & $\sqrt{ }$ \\
\hline [10] & $\begin{array}{l}\text { Beam divergence } \\
\text { effects on WDM-FSO } \\
\text { system }\end{array}$ & $\begin{array}{c}\text { WDM- } \\
\text { FSO model }\end{array}$ & $\sqrt{ }$ & $\sqrt{ }$ & & $\sqrt{ }$ & $\sqrt{ }$ \\
\hline [11] & $\begin{array}{l}\text { Modulation formats \& } \\
\text { weather effects on FSO }\end{array}$ & $\begin{array}{l}\text { RZ, NRZ, } \\
\text { raised } \\
\text { cosine } \\
\text { encoding }\end{array}$ & & $\sqrt{ }$ & $\sqrt{ }$ & $\sqrt{ }$ & \\
\hline [12] & $\begin{array}{l}\text { Analysis of chaotic } \\
\text { FSO system under } \\
\text { weather effects }\end{array}$ & \begin{tabular}{|c|} 
Chaotic \\
FSO model
\end{tabular} & & $\sqrt{ }$ & $\sqrt{ }$ & $\sqrt{ }$ & \\
\hline [13] & $\begin{array}{l}\text { Optimization of FSO } \\
\text { related parameters }\end{array}$ & $\begin{array}{c}\text { Link } \\
\text { margin } \\
\text { analysis }\end{array}$ & $\sqrt{ }$ & & $\sqrt{ }$ & $\sqrt{ }$ & \\
\hline [14] & $\begin{array}{c}\text { Performance of } \\
\text { 120Gbps DP-16-QAM } \\
\text { FSO link under weather } \\
\text { effects }\end{array}$ & $\begin{array}{l}\text { DP-16- } \\
\text { QAM }\end{array}$ & & $\sqrt{ }$ & $\sqrt{ }$ & $\sqrt{ }$ & \\
\hline [15] & $\begin{array}{c}\text { Comparison of QPSK } \\
\text { modulation techniques } \\
\text { for FSO link }\end{array}$ & $\begin{array}{c}16- \\
\text { QAM,64- } \\
\text { QAM }\end{array}$ & & & $\sqrt{ }$ & $\sqrt{ }$ & \\
\hline [16] & $\begin{array}{c}\text { FSO based ROF-WDM } \\
\text { system analysis with } \\
\text { advance modulation } \\
\text { formats under } \\
\text { atmospheric effects }\end{array}$ & $\begin{array}{l}\text { CSRZ, } \\
\text { DB, AMI }\end{array}$ & $\sqrt{ }$ & $\sqrt{ }$ & $\sqrt{ }$ & $\sqrt{ }$ & \\
\hline [17] & $\begin{array}{l}\text { Performance of FSO } \\
\text { link based on weather } \\
\text { conditions }\end{array}$ & \begin{tabular}{|c|} 
Kim, \\
Kruse and \\
Al Nabulsi \\
model
\end{tabular} & & $\sqrt{ }$ & $\sqrt{ }$ & $\sqrt{ }$ & \\
\hline [18] & $\begin{array}{l}\text { Transmitting a 1.28- } \\
\text { Tb/s (32 } 40 \mathrm{~Gb} / \mathrm{s}) \\
\text { (WDM) signal over } \\
\text { FSO link }\end{array}$ & WDM & $\sqrt{ }$ & & & $\sqrt{ }$ & \\
\hline [19] & $\begin{array}{c}\text { High capacity } 1.28 \mathrm{Tbps} \\
\text { multiplexed FSO } \\
\text { system using QPSK } \\
\text { modulation }\end{array}$ & \begin{tabular}{|} 
QPSK, RZ, \\
NRZ
\end{tabular} & $\sqrt{ }$ & & $\sqrt{ }$ & $\sqrt{ }$ & \\
\hline [20] & $\begin{array}{c}\text { Performance analysis of } \\
\text { FSO system for S, C } \\
\text { and L band }\end{array}$ & RZ, NRZ & $\sqrt{ }$ & $\sqrt{ }$ & $\sqrt{ }$ & $\sqrt{ }$ & \\
\hline [21] & $\begin{array}{c}\text { Performance } \\
\text { enhancement for dual- } \\
\text { polarized DWDM- } \\
\text { OFDM FSO system }\end{array}$ & CO-OFDM & $\sqrt{ }$ & $\sqrt{ }$ & $\sqrt{ }$ & $\sqrt{ }$ & $\sqrt{ }$ \\
\hline [22] & $\begin{array}{l}\text { A comprehensive } \\
\text { survey on FSO } \\
\text { communication }\end{array}$ & $\begin{array}{l}\text { PPM, } \\
\text { OOK }\end{array}$ & & & $\sqrt{ }$ & $\sqrt{ }$ & \\
\hline
\end{tabular}

\section{Contributions}

In this paper, we have implemented 4-channel DWDM on the FSO channel with equal spacing over 760-850 nm band. Performance evaluation of proposed system is carried out by considering various parameters. Performance comparison based on these parameters include:

- Data rate vs Q-factor

- $\quad$ FSO link length vs BER

- Transmitter power vs Q-factor

- Amplifier gain vs Q-factor

- $\quad$ FSO length vs Q-factor

- Q-factors in different weather conditions

- Comparison of C, L and proposed band

Our results are evaluated in licensed version of Optisystem 14.0 and MATLAB 2016a. The rest of the paper is organized as follows. Section II comprises mathematical modeling whereas proposed model of DWDM based FSO system is given in Section III, followed by results and discussions in Section IV. Finally, the paper is concluded in Section V.

\section{MATHEMATICAL MODEL}

This section investigates the link budget analysis in FSO based communication system including the transmission range and communication link model and provides a study on environmental phenomena such as rain, fog, and haze that affects smooth transmission in FSO links.

\section{A. Weather Attenuation Effects}

The type of attenuation that occurs due to the presence of aerosols is termed as atmospheric attenuation. The cause of this attenuation is due to the additive effect of absorption and scattering of infrared light by gas molecules in the atmosphere.

The atmospheric loss can be expressed in $\mathrm{dB}$ as (Fadhil et al., 2013)

$\operatorname{Loss}_{\text {propagation }}=-10 \log _{10} \mathrm{~T}_{\mathrm{a}}$

Where:

$\mathrm{T}_{\mathrm{a}}=$ The ratio of power received to the power transmitted into the optical link and named as atmospheric transmittance.

i. Rain Attenuation:

Rain attenuation occurs when the raindrops of large enough size cause reflection and refraction of optical signals. The attenuation is expressed in $\mathrm{dB} / \mathrm{km}$ and calculated as [7]:

$\mathrm{Att}_{\mathrm{rain}}=\mathrm{k}_{1} \mathrm{R}^{\mathrm{k}_{2}}$

Where:

$\mathrm{R}=$ Represents rain rate in $\mathrm{mm} / \mathrm{hr}$.

$\mathrm{k}_{1}$ and $\mathrm{k}_{2}=$ Are model parameters that depend upon raindrop size and rain temperature.

The value of $\mathrm{R}$ according to different rain conditions is shown in the table i.e., Table III below.

Table III: R Values for Rain Conditions

\begin{tabular}{|l|c|}
\hline \multicolumn{1}{|c|}{ Precipitation } & R Values $(\mathbf{m m} / \mathbf{h r})$ \\
\hline Light rain & 2.5 \\
\hline Medium rain & 12.5 \\
\hline Heavy rain & 25 \\
\hline Cloud burst \& heavy rain & 100 \\
\hline
\end{tabular}

ii. Haze Attenuation:

Atmospheric attenuation caused by haze can be expressed using Beer-Lambert law as [16]:

$\propto=\mathrm{e}^{-\sigma l}$

Where:

$\sigma=$ The attenuation coefficient per unit length and $1=$ The distance between transmitter and receiver.

The value of $\sigma$ can be calculated using Kruse relation given as [6]: 
$\boldsymbol{\sigma} \cong \frac{3.912}{\mathrm{~V}}\left(\frac{\lambda}{550}\right)^{-\mathrm{q}}$

Where:

$\mathrm{V}=$ Represents visibility in $\mathrm{km}$,

$\lambda=$ The wavelength in $\mathrm{nm}$ and

$\mathrm{q}=$ Represents the size distribution of diffusing particles Atmospheric attenuation due to scattering and absorption of light can be found using Beer's law as [16]:

$\mathrm{I}=\mathrm{I}_{0} \mathrm{e}^{(-\gamma \mathrm{x})}$

Where:

$\gamma=$ The attenuation coefficient and

$\mathrm{I}$ and $\mathrm{I}_{0}=$ Detected and initial intensities at certain locations $\mathrm{x}$

iii. Fog attenuation:

Fog is one of the most critical attenuation factors among all other conditions in FSO communication. Mie scattering theory is the most accurate procedure to calculate attenuation in fog [9]. However, it demands much detailed information of fog parameters such as particle size, size distribution etc., which cannot be readily available at the location of installation and requires complex calculations. To calculate fog attenuation, Kruse, Kim, and Al-Nabulsi use the above approach and predict attenuation using visibility. Kim model rejects the wavelength-dependent attenuation for visibility in dense fog as mentioned in equation (4). The value of the q variable for the Kim model is given as:

$\mathbf{q}=\left\{\begin{array}{cr}1.6 & \text { if } \mathbf{V}>\mathbf{5 0 K m} \\ 1.3 & \text { if } 6 \mathrm{Km}<\mathrm{V}<50 \mathrm{Km} \\ 0.16 \mathrm{~V}+1.344 & \text { if } 1 \mathrm{Km}<\mathrm{V}<6 \mathrm{Km} \\ \mathrm{V}-05 & \text { if } 0.5 \mathrm{Km}<\mathrm{V}<1 \mathrm{Km} \\ 0 & \text { if } \mathrm{V}<0.5 \mathrm{Km}\end{array}\right\}$

This model explains that higher wavelengths will face less attenuation e.g. $1550 \mathrm{~nm}$ will have less attenuation than shorter wavelengths.

Parameter "q" as given by Kruse model is listed below i.e., Table IV [12].

Table IV: Size Distribution of Scattering Particles

\begin{tabular}{|l|c|c|}
\hline \multicolumn{1}{|c|}{ Visibility Type } & $\mathbf{q}$ & Visibility Length $(\mathbf{k m})$ \\
\hline Low & 0.585 & $\mathrm{~V}<6$ \\
\hline Average & 1.3 & $6<\mathrm{V}<50$ \\
\hline Very High & 1.6 & $\mathrm{~V}>50$ \\
\hline
\end{tabular}

\section{B. Link Margin}

Link margin provides the threshold for the link performance and it is an important parameter to be evaluated before attenuation that affects the link. Link margin also termed as the fade margin, is the percentage of time the link functions satisfactorily. When the link margin does not exceed its limit, is termed as link availability. We can calculate the link margin as follows [6]:

$\mathrm{M}_{\text {Link }}(\mathrm{dB})=\mathrm{P}_{\mathrm{e}}+\left|\mathrm{S}_{\gamma}\right|-\mathrm{Att}_{\text {geo }}(\mathrm{dB})-\mathrm{Att}_{\mathrm{mol}}-\mathrm{P}_{\text {total }}$

(7)
Where:

$\mathrm{P}_{\mathrm{e}}=$ The total power of the transmitter in $\mathrm{dB}$,

$\mathrm{S}_{\gamma}=$ Receiver sensitivity in $\mathrm{dBm}$

$\mathrm{Att}_{\mathrm{geo}}=$ Geometrical attenuation in $\mathrm{dB}$

$\mathrm{Att}_{\mathrm{mol}}=$ Molecular attenuation in $\mathrm{dB}$ and

$\mathrm{P}_{\text {total }}=$ System losses

Link margin can also be calculated by using the equation below [16]:

$\mathrm{LM}=10 \log \frac{\mathrm{P}_{\mathrm{R}}}{\mathrm{S}_{\gamma}}$

For the signal to be detected at the receiver end, its power should be greater than the receiver sensitivity. So, received signal power should be calculated for quality check in FSO communication.

\section{Minimum Transmission Range}

The transmittivity is defined in terms of absorbance $\sigma$ as [4]:

$$
\begin{aligned}
\mathrm{F}_{\mathrm{l}} & =\left(-10 \log \left(\frac{\mathrm{I}_{1}}{\mathrm{I}_{0}}\right)\right)=10 \log \left(\frac{\mathrm{p}_{\text {receiver }}}{\mathrm{p}_{\text {total }}}\right) \\
& =-10 \log \left(\mathrm{e}^{-\sigma \mathrm{l}}\right)
\end{aligned}
$$

Where:

$\mathrm{F}_{1}=$ The attenuation in $\mathrm{dB}$

$\mathrm{P}_{\text {total }}=$ Total power transmitted in watts while,

$\mathrm{P}_{\text {receiver }}=$ Received power in watts.

\section{Receiver Signal Power}

To calculate the signal power at the receiver, consider a laser is transmitting the signal at power of $\mathrm{P}_{\text {trans. }}$ At the detector, the signal power received can be expressed as [9].

$\mathrm{P}_{\text {Receiver }}=\mathrm{P}_{\text {Trans }} \frac{\mathrm{D}^{2}}{\vartheta_{\text {div }}^{2} \mathrm{~L}^{2}} \cdot 10^{\frac{-\gamma \mathrm{L}}{10}} \tau_{\text {trans }} \tau_{\text {received }}$

Where:

$\mathrm{D}=$ Represents receiver diameter

$\theta=$ Divergence angle

$\gamma=$ The attenuation factor while

$\tau_{\text {trans }}$ and $r_{\text {eceived }}=$ The optical efficiency of transmitter and receiver

\section{E. Data Rate}

To evaluate the data rate, we consider a laser with transmitter power $P_{\text {trans }}$ and transmitter divergence $\theta$, the achievable data rate can be calculated as [9]:

$$
R=\frac{P_{T} \tau_{\text {trans }} \tau_{\text {rec }} 10^{-\frac{\gamma \cdot \mathrm{L}}{10}} D^{2}}{\pi(\theta \mid 2)^{2} L^{2} E_{p} N_{b}}
$$

Which can also be written as:

$\mathrm{R}=\frac{4}{\pi \mathrm{E}_{\mathrm{p}} \mathrm{N}_{\mathrm{b}}} \cdot \mathrm{P}_{\mathrm{rec}}$

Where:

$E_{p}=h c / \lambda$ is the energy of photon at a given wavelength 


\section{SYSTEM DESIGN}

The proposed 4-channel DWDM based FSO communication model is presented in Fig. 1. It consists of a transmitter, multiplexer, free space medium, optical amplifier, demultiplexer, and receiver. The data source, $\mathrm{CW}$ laser, pulse generator and Mach-Zehnder modulator combine to form a transmitter. consists of PIN photodetector, low pass Gaussian filter, (that reduces the sharpness of received signal), eye diagram analyzers and spectrum analyzer to study the signal. Before it reaches the receiver, the OA amplifies the received signal and sends it to $1 \times 4$ WDM DEMUX, out of which multiplexed signal divides into four streams.

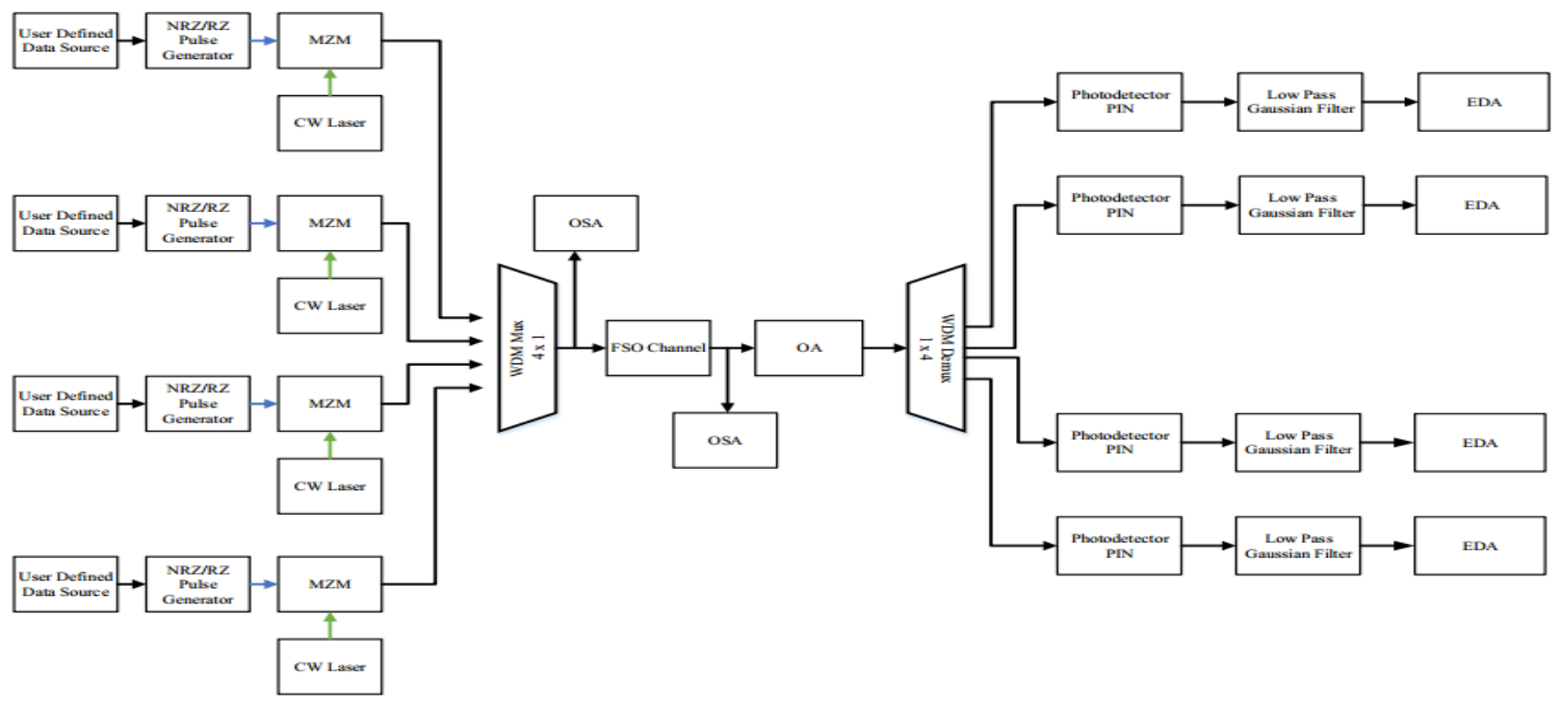

Fig. 1: Four-Channel DWDM Based FSO Communication Model

Data bits generated by PRBS are fed to a pulse generator to form pulses, which are then transferred to the modulator. The function of the pulse generator is to convert the logical signal into an electrical signal to pass it to the modulator. The modulator receives two inputs i.e. electrical signal from the pulse generator and a carrier signal from $\mathrm{CW}$ laser. The continuous-wave laser source operates at each transmitter frequency and power of $20 \mathrm{dBm}$. The primary function of the modulator is to convert the electrical signal into an optical signal as a medium is a free space, see Table V.

Table V: Operating System Parameters

\begin{tabular}{|l|c|}
\hline \multicolumn{2}{|c|}{ Parameters } \\
\\
\hline Operating wavelength & Value \\
\hline Data rate & $760-850 \mathrm{~nm}$ \\
\hline CW laser Power & $1 \mathrm{Gbps}$ \\
\hline FSO link range & $20 \mathrm{dBm}$ \\
\hline Attenuation & $1-4 \mathrm{~km}$ \\
\hline Filter & $20 \mathrm{~dB} / \mathrm{km}$ \\
\hline Amplifier Gain & Low pass Gaussian \\
\hline Channel Spacing & $20 \mathrm{~dB}$ \\
\hline Modulation Scheme & Equal \\
\hline
\end{tabular}

The four transmitters are operating on equal channel spaced frequency in the basic design i.e. $760 \mathrm{~nm}, 765 \mathrm{~nm}, 770 \mathrm{~nm}, 775$ $\mathrm{nm}$. The modulated data from all transmitters are forward to $4 \times 1$ WDM MUX which multiplexes the signals and transmits over the FSO channel. OSA is used to check the signal power of the signal before and after the FSO link. The receiver
The signal reaches the photodetector after passing through the FSO channel and demultiplexer. The photodetector converts the received optical signal to an electrical signal and passes it through a LPF. Now, the signal is filtered to remove undesired components from the desired electrical signal. EDA is used to check the signal quality and noise margins of the data signal. Receiver parameters include receiver sensitivity of $-18 \mathrm{dBm}$, the responsivity of $1 \mathrm{~A} / \mathrm{W}$ and dark current of $10 \mathrm{nA}$.

Table VI: Tx \& Rx Parameters

\begin{tabular}{|l|c|c|}
\hline \multicolumn{1}{|c|}{ Parameters } & Transmitter & Receiver \\
\hline Wavelength $(\mathrm{nm})$ & $760,765,770,775$ & $760,765,770,775$ \\
\hline Power & $20 \mathrm{dBm}$ & - \\
\hline Aperture diameter & $1 \mathrm{~m}$ & $10 \mathrm{~m}$ \\
\hline Sensitivity & - & $-18 \mathrm{dBm}$ \\
\hline Responsivity & - & $1 \mathrm{~A} / \mathrm{W}$ \\
\hline Dark current & - & $10 \mathrm{nA}$ \\
\hline
\end{tabular}

The errors in the output signal can be determined using the BER analyzer and eye diagram analyzer. Transmitter and receiver parameters are mentioned in Table VI. The system is encoded using RZ/NRZ scheme.

The amplifier gain is $20 \mathrm{~dB}$ and the FSO channel parameters include distance of $2.5 \mathrm{~km}$ in the generic model, the transmitter aperture diameter of $1 \mathrm{~m}$, the receiver aperture diameter of $10 \mathrm{~m}$, the data rate of $1 \mathrm{Gbps}$ and attenuation of 20 $\mathrm{dB} / \mathrm{km}$. 


\section{RESULTS AND DISCUSSION}

The results of proposed scheme are presented in different sections as follow:

\section{A. Data rate vs $Q$-factor}

The comparison of data rates and Q-factors obtained against four channels of WDM is shown in Fig. 2. Overall high Qfactors show that simulations are made to receive good quality signal at the receiver end. Fig. 2 depicts that with the increase in data rate, the Q-factor decreases. All four channels have almost same response towards increasing data rates. It is observed that Q-factor reaches a peak at low data rates. It means that at low data rates, the highest quality can be achieved at the receiver. However, trade-off exists between data rates and distances. Increasing data rates means limiting the distances. Further, due to DWDM significant data rate is increased due to four channels as compared to [12] in which only single channel is used to achieve total data rate of $2.5 \mathrm{~Gb} / \mathrm{sec}$.

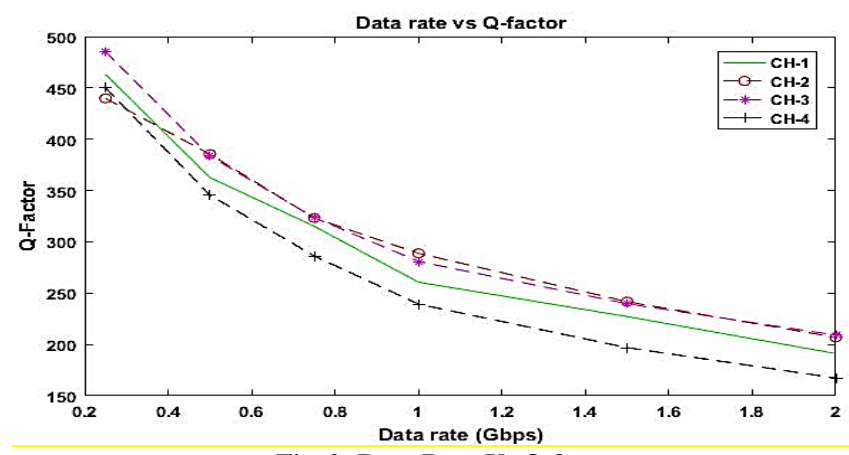

Fig. 2: Data Rate Vs Q-factor

\section{B. FSO Link Length Vs BER}

Fig. 3 shows comparison of FSO link length and BER. BER is plotted on the logarithmic scale. Graph shows that channels with less frequency have a low BER but as the distance increases, the trend becomes the same for all channels. At large distances, the BER approaches to 1.0 which indicates that significant errors will be seen at larger distances.

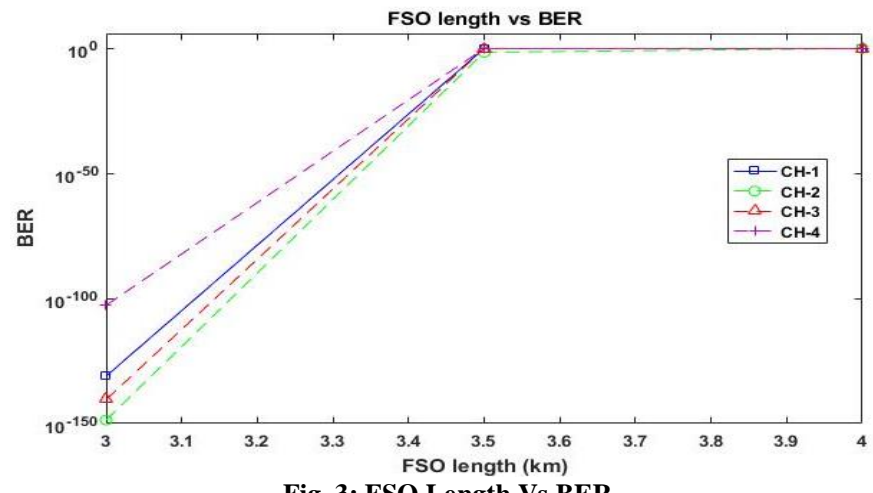

Fig. 3: FSO Length Vs BER

However, lengths having low BER can be used in access side networks, military applications and for interconnectivity between LANs.

\section{Transmitter Power Vs Q-factor}

The comparison between transmitter power and Q-factor is shown in Fig. 4. When the transmitted power is increased gradually, the Q-factor increases dominantly for all four channels. The low power levels correspond to the low Q-factor. Hence, for achieving high Q-factor, the transmitter power is an efficient parameter to use.

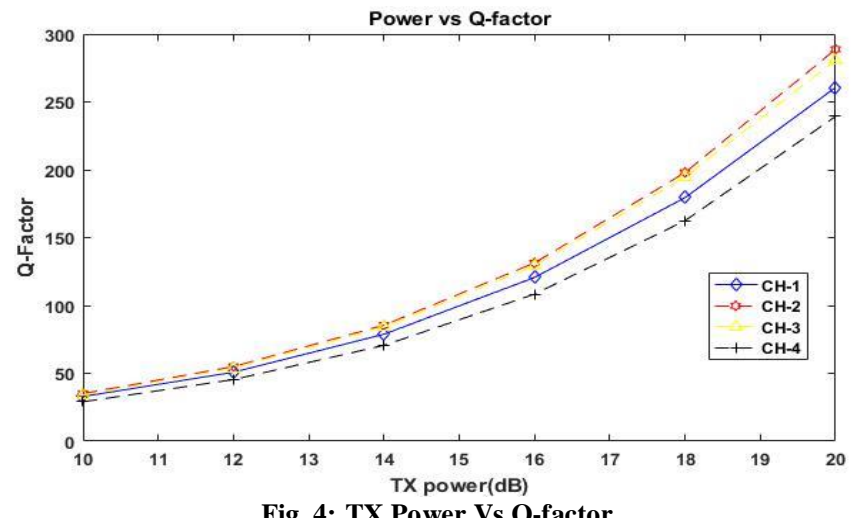

\section{Amplifier Gain Vs Q-factor}

Amplifier gain has a similar effect as above. As the gain increases the Q-factor also increases while at low gain, the Qfactor does not rise much. So, the amplifier gain can also be used to increase the Q-factor. The illustration is given in Fig. 5.

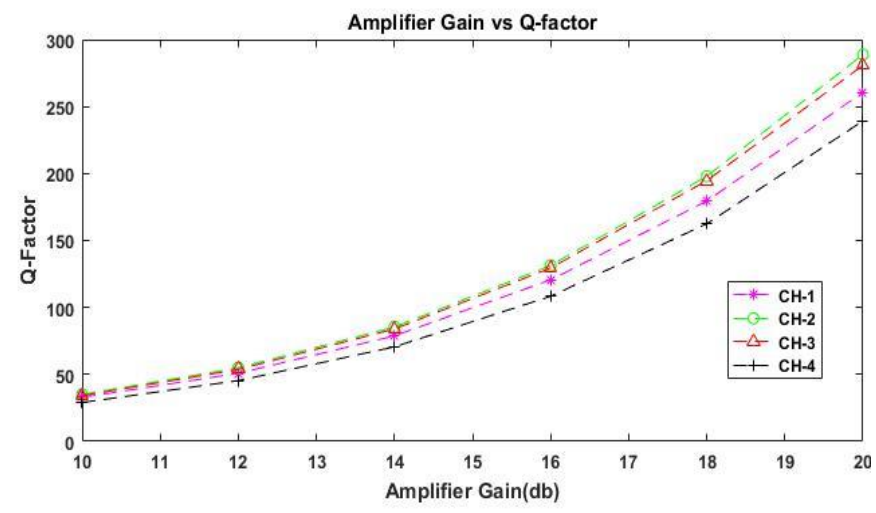

Fig. 5: Amplifier Gain Vs Q-factor

\section{E. FSO Length Vs Q-factor}

As depicted in Fig.6, the FSO link length shows a different behavior for Q-factor. Initially, when the distance is set up to 2 $\mathrm{km}$, the Q-factor is very good but as the distance reaches to 3 $\mathrm{km}$, it falls off gradually and above $3 \mathrm{~km}$ it approaches to zero. So, till $2 \mathrm{~km}$ of range good Q-factor can be attained.

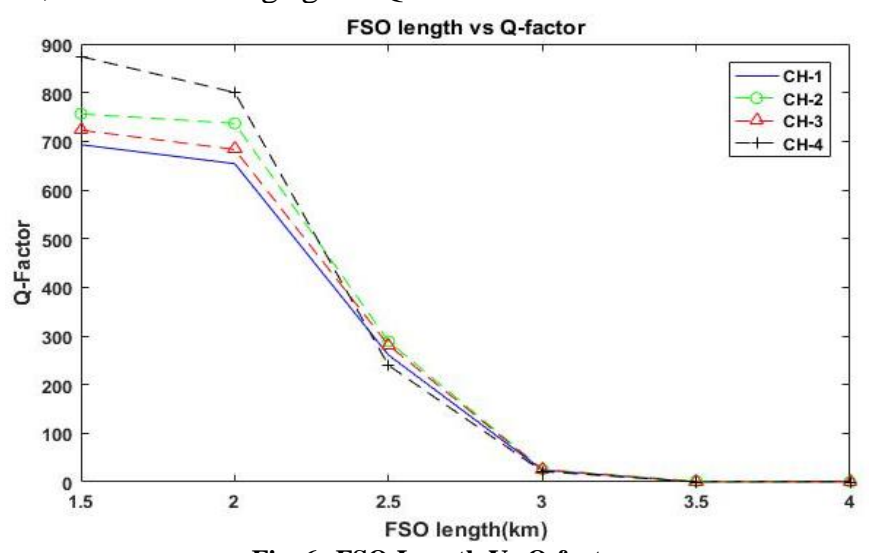

Fig. 6: FSO Length Vs Q-factor. 


\section{F. Attenuation Vs Weather Conditions}

Fig. 7 illustrates the effect of weather conditions and attenuation on the Q-factors. Weather conditions include light rain, heavy rain, little fog, moderate fog, and haze. Light rain and little fog behave alike. They show high Q-factor against all wavelengths. The moderate fog shows relatively less Q-factor but higher than heavy rain and haze. It can be seen that haze has the lowest Qfactor while heavy rain has a relatively higher Q-factor than haze. So, weather conditions affect Q-factor in different ways. Attenuation values against different weather are shown in Table VII.

Table VII: Attenuation for Different Weathers.

\begin{tabular}{|l|c|}
\hline \multicolumn{1}{|c|}{ Weather Conditions } & Attenuation, $\mathbf{d B} / \mathbf{k m}$ \\
\hline Light Rain & 6.2702 \\
\hline Heavy Rain & 19.795 \\
\hline Little Fog & 4.2850 \\
\hline Moderate Fog & 15.555 \\
\hline Haze & 20.68553 \\
\hline
\end{tabular}

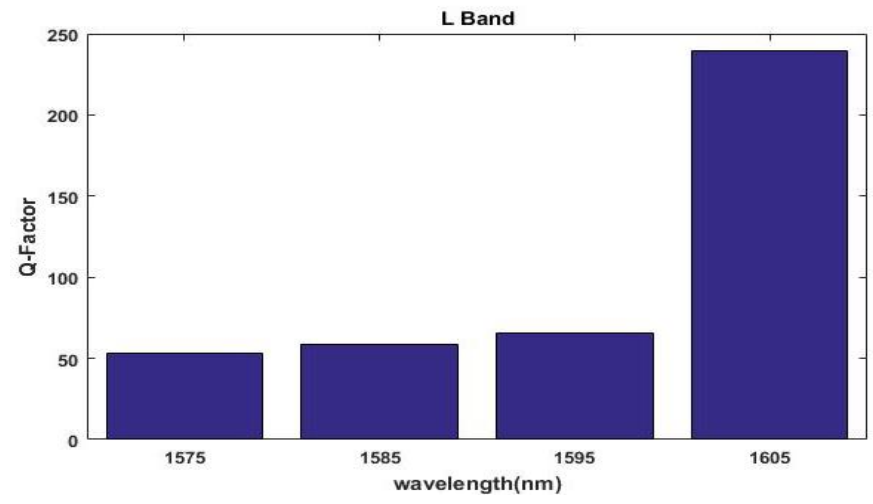

Fig. 7: Weather Conditions Vs Q-factor

\section{G. Comparison of 760-850nm, $C \&$ \& Bands}

We have shown a comparison of $\mathrm{C}$, L-band wavelengths with $760-850 \mathrm{~nm}$ band which we propose in our design. C-band operates in 1530-1560 $\mathrm{nm}$ and L-band operates in 1575-1620 $\mathrm{nm}$. The comparison of each band in terms of Q-factor is given in Fig. 8, 9 and 10.

It can be seen in Fig. 8 that a low wavelength band of 760-850 $\mathrm{nm}$ gives high Q-factors. It means that in FSO communication wavelengths should near infrared range for achieving good results. Fig. 9 shows the performance of C-band. The highest Qfactor is observed at $1530 \mathrm{~nm}$ while it decreases gradually as the wavelength increases. But these Q-factors are less than those which are used in previous band i.e. from $760-850 \mathrm{~nm}$.

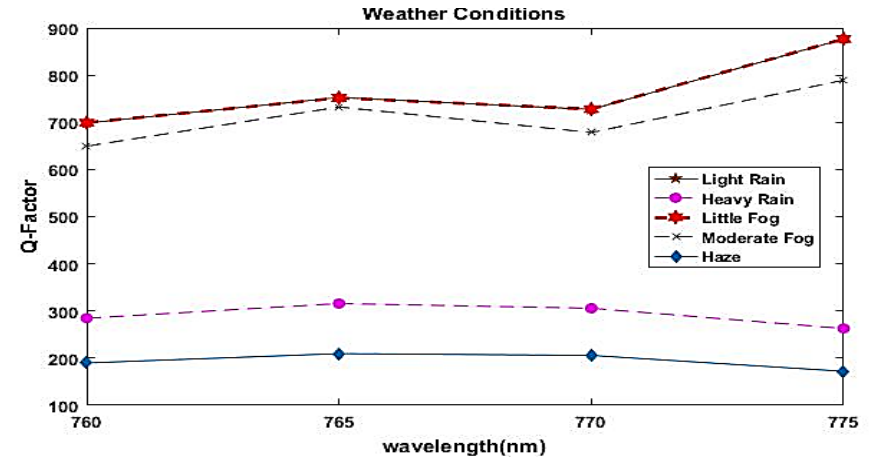

Fig. 8: Q-factors in 760-850 $\mathrm{nm}$ Band

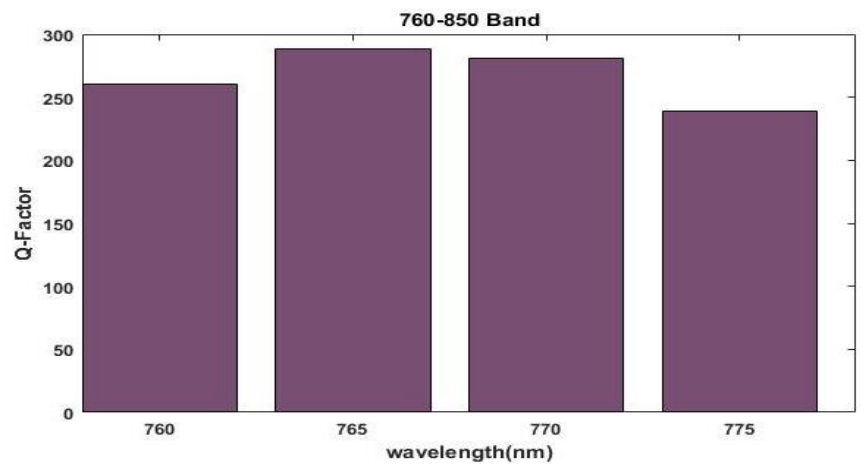

Fig. 9: Q-factors in C-band Operation

Fig.10 shows the performance of L-band in terms of Q-factors. At $1575 \mathrm{~nm}$ of wavelength, the Q-factor decreases but in the similar band highest Q-factor is observed at $1605 \mathrm{~nm}$.

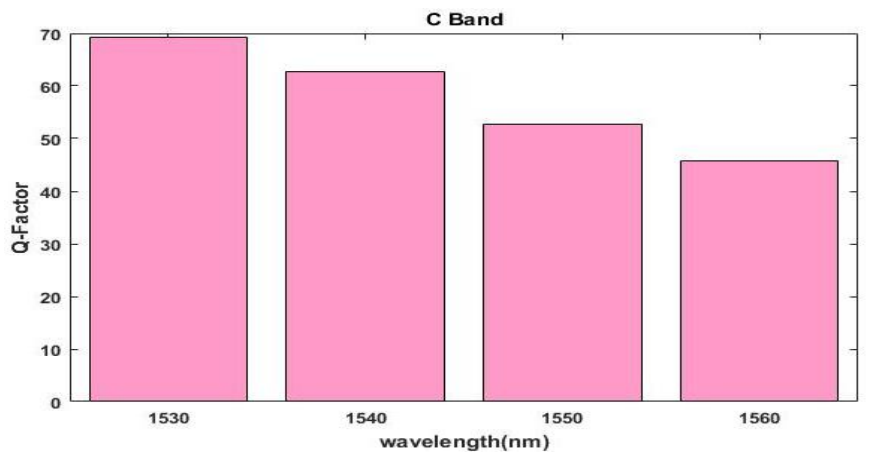

Fig. 10: Q-factors in L-band Operation

\section{CONCLUSION}

The paper is composed of two parts. In the first part, the advantages, challenges, applications, and related contribution done in the field of FSO communication were demonstrated. A brief comparison of optical fiber and FSO communication is added in this section to show the effectiveness of FSO model in the situations where optical fiber cannot be used. Role of FSO communication in current and evolving networks is also highlighted in the first section. In the second part, four-channel DWDM based FSO communication were implemented. Equal channel spacing is considered for the working of this model. While performing simulations, different parameters are analyzed to evaluate the performance of proposed model. These parameters include data rate, Q-factor, FSO link length, BER, transmitter power, amplifier gain, attenuation and weather conditions. Proposed model is tested for different wavelengths band, operating at different distances upto $5 \mathrm{~km}$. While comparing the performance of wavelength band from $760 \mathrm{~nm}$ to $775 \mathrm{~nm}$, systems performs exceptionally well as compared to its working in $\mathrm{C}$ and $\mathrm{L}$ band. By increasing transmitter power and amplifier gain, significant improvement is seen in results. The proposed model when tested under different weather conditions, show that haze is the worst weather condition for the working of FSO model. 


\section{ACKNOWLEDGMENT}

This research work is financially supported by the ASR \& TD department of UET TAXILA, under grant no. UET/ASR\&TD/RG-357.

\section{REFERENCES}

[1] Kaur, A., \& Sheetal, A. (2015). Performance analysis of $16 \times 2.5$ $\mathrm{GB} / \mathrm{s}$ FSO system for the most critical weather conditions. Int $\mathrm{J}$ Adv Res Comput Commun Eng, 4(5), 612-618.

[2] Al Ghadhban, A., Celik, A., Shihada, B., \& Alouini, M.-S. (2019). LightFDG: An Integrated Approach to Flow Detection and Grooming in Optical Wireless DCNs. IEEE Transactions on Network and Service Management.

[3] Ansari, I. S., Alouini, M.-S., \& Yilmaz, F. (2013). On the performance of hybrid RF and RF/FSO fixed gain dual-hop transmission systems. Paper presented at the 2013 Saudi International Electronics, Communications and Photonics Conference.

[4] Beshr, M., \& Aly, M. H. (2015). Outdoor wireless optical communication system attenuation at different weather conditions. International Journal of Optics, 2015, 2015.

[5] Nadeem, F., Kvicera, V., Awan, M. S., Leitgeb, E., Muhammad, S. S., \& Kandus, G. (2009). Weather effects on hybrid FSO/RF communication link. IEEE journal on selected areas in communications, 27(9), 1687-1697.

[6] Zabidi, S. A., Al Khateeb, W., Islam, M. R., \& Naji, A. (2010). The effect of weather on free space optics communication (FSO) under tropical weather conditions and a proposed setup for measurement. Paper presented at the International Conference on Computer and Communication Engineering (ICCCE'10).

[7] Vavoulas, A., Sandalidis, H. G., \& Varoutas, D. (2012). Weather effects on FSO network connectivity. IEEE/OSA Journal of Optical Communications and Networking, 4(10), 734-740.

[8] Malik, A., \& Singh, P. (2015). Comparative analysis of point to point FSO system under clear and haze weather conditions. Wireless personal communications, 80(2), 483-492.

[9] Jung, K.-J., Nam, S. S., Alouini, M.-S., \& Ko, Y.-C. (2019). Unified Statistical Channel Model of Ship (or Shore)-to-Ship FSO Communications with Pointing Errors. Paper presented at the 2019 IEEE Conference on Standards for Communications and Networking (CSCN).

[10] Chaudhary, S., Bansal, P., \& Lumb, M. (2014). Effect of beam divergence on WDM-FSO transmission system. International Journal of Computer Applications, 93(1).

[11] Chaudhary, S., Bansal, P., \& Singh, G. (2013). Implementation of FSO network under the Impact of atmospheric turbulences. International Journal of Computer Applications, 75(1).

[12] Niaz, A., Qamar, F., Ali, M., Farhan, R., \& Islam, M. K. (2019). Performance analysis of chaotic FSO communication system under different weather conditions. Transactions on Emerging Telecommunications Technologies, 30(2), e3486.

[13] Fadhil, H. A., Amphawan, A., Shamsuddin, H. A., Abd, T. H., AlKhafaji, H. M., Aljunid, S., \& Ahmed, N. (2013). Optimization of free space optics parameters: An optimum solution for bad weather conditions. Optik, 124(19), 3969-3973.

[14] Kakati, D., \& Arya, S. C. (2019). Performance of 120 Gbps single channel coherent DP-16-QAM in terrestrial FSO link under different weather conditions. Optik, 178, 1230-1239.

[15] Kadhim, L. A. (2014). 16/64qam modulation technique for free space optical communication system. International Journal of Advancements in Computing Technology, 6(6), 1.

[16] Singh, P., \& Kaur, E. H. (2018). Analysis of FSO based ROFWDM System with Advance Intensity Modulation Techniques Under Various Atmospheric Conditions. Paper presented at the 2018 Second International Conference on Electronics, Communication and Aerospace Technology (ICECA).

[17] Hameed, N., Mehmood, T., \& Manzoor, H. U. (2017). Effect of Weather Conditions on FSO link based in Islamabad. arXiv preprint arXiv:1711.10869.
[18] Ciaramella, E., Arimoto, Y., Contestabile, G., Presi, M., D'errico, A., Guarino, V., \& Matsumoto, M. (2009). 1.28-Tb/s (32\$ltimes $\$ 40 \mathrm{~Gb} / \mathrm{s})$ Free-Space Optical WDM Transmission System. IEEE Photonics Technology Letters, 21(16), 1121-1123.

[19] Patnaik, B., \& Sahu, P. (2012). Novel QPSK modulation for DWDM free space optical communication system. Paper presented at the 2012 Wireless Advanced (WiAd).

[20] Jain, D., \& Mehra, R. (2017). Performance analysis of Free Space Optical communication system for S, C and L band. Paper presented at the 2017 International Conference on Computer, Communications and Electronics (Comptelix).

[21] Mohamed, A. E.-N. A., Rashed, A. N. Z., Tabbour, M. S., \& Ismail, A. M. (2017). Performance enhancement of free space optical (FSO) systems using polarized DWDM-OFDM technique under atmospheric disturbances. Paper presented at the 2017 13th International Computer Engineering Conference (ICENCO).

[22] Khalighi, M. A., \& Uysal, M. (2014). Survey on free space optical communication: A communication theory perspective. IEEE communications surveys \& tutorials, 16(4), 2231-2258. 